\title{
An Automated Real-Time Localization System in Highway and Tunnel Using UWB DL-TDoA Technology
}

\author{
Long Wen, ${ }^{1}$ Jinkun Han $\mathbb{D}^{2},{ }^{2}$ Liangliang Song ${ }^{\circ},{ }^{3}$ Qi Zhang, ${ }^{4}$ Kai Li, ${ }^{1}$ Zhi Li, ${ }^{4}$ Weimin Zhang, ${ }^{5}$ \\ Beihai Zhang, ${ }^{5}$ Xin You, ${ }^{5}$ Yunsick Sung, ${ }^{6}$ Sumi Ji, ${ }^{6}$ and Wei Song ${ }^{7}$
}

${ }^{1}$ Beijing Municipal Engineering Research, Beijing, China

${ }^{2}$ Department of Computer Science, Georgia State University, Atlanta, 30303 GA, USA

${ }^{3}$ Roadway Smart (Beijing) Technology Co., Ltd., Beijing, China

${ }^{4}$ Beijing Capital Road Development Group Co., LTD., Beijing, China

${ }^{5}$ Beijing Sutong Technology Co., Ltd., Beijing, China

${ }^{6}$ Department of Multimedia Engineering, Dongguk University-Seoul, Seoul 04620, Republic of Korea

${ }^{7}$ School of Information Science and Technology, North China University of Technology, Beijing 100144, China

Correspondence should be addressed to Jinkun Han; hjinkun1@student.gsu.edu

and Liangliang Song; liangliang.song@roadwaysmart.com

Received 8 September 2020; Revised 17 October 2020; Accepted 30 October 2020; Published 23 November 2020

Academic Editor: Shaohua Wan

Copyright (c) 2020 Long Wen et al. This is an open access article distributed under the Creative Commons Attribution License, which permits unrestricted use, distribution, and reproduction in any medium, provided the original work is properly cited.

There exists an electromagnetic shielding effect on radio signals in a tunnel, which results in no satellite positioning signal in the tunnel scenario. Moreover, because vehicles always drive at a high speed on the highway, the real-time localization system (RTLS) has a bottleneck in a highway scenario. Thus, the navigation and positioning service in tunnel and highway is an important technology difficulty in the construction of a smart transportation system. In this paper, a new technology combined downlink time difference of arrival (DL-TDoA) is proposed to realize precise and automated RTLS in tunnel and highway scenarios. The DL-TDoA inherits ultra-wideband (UWB) technology to measure the time difference of radio signal propagation between the location tag and four different location base stations, to obtain the distance differences between the location tag and four groups of location base stations. The proposed solution achieves a higher positioning efficiency and positioning capacity to achieve dynamic RTLS. The DL-TDoA technology based on UWB has several advantages in precise positioning and navigation, such as positioning accuracy, security, anti-interference, and power consumption. In the final experiments on both static and dynamic tests, DL-TDoA represents high accuracy and the mean errors of $11.96 \mathrm{~cm}, 37.11 \mathrm{~cm}, 50.06 \mathrm{~cm}$, and $87.03 \mathrm{~cm}$ in the scenarios of static tests and $30 \mathrm{~km} / \mathrm{h}, 60 \mathrm{~km} / \mathrm{h}$, and $80 \mathrm{~km} / \mathrm{h}$ in dynamic tests, respectively, which satisfy the requirements of RTLS.

\section{Introduction}

Smart highway integrates and applies advanced information processing technology, sensing technology, and transmission technology $[1,2]$. These positioning technologies form an open and common basic platform for monitoring vehicles. Smart highway are aimed at being efficient, convenient, safe, and green, combined with various open operation management and service modes [3], and it provides reliable traffic network service $[4,5]$ for the rapid transportation of people and goods, provides free communication pipeline service for the vehicle to vehicle [6] or vehicle to road interaction, provides full-time responsive emergency service for emergencies, and provides refined and independent travel service for travelers.

The tunnel project shortens the road distance and reduces the sloping road, so it significantly improves the traffic capacity. Due to the natural electromagnetic shielding effect of the tunnel on radio signals, there is no satellite positioning signal in the tunnel. Therefore, the navigation and positioning service in the tunnel has always been an important problem in the construction of a smart highway. To actively respond to the ideas and development strategy of smart highway construction and improve the industry 
localization service, a new solution should be proposed to solve the problem of navigation and positioning service in the highway and tunnel without changing the terminals or increasing the complexity of terminals.

Positioning technology in highway and tunnel is a recognized difficulty in both the industry and academia. The existing positioning technologies mainly contain Bluetooth $[7,8]$, Wi-Fi [9], Radio Frequency Identification (RFID) [10], ZigBee [11], ultra-wideband (UWB) [12-14], infrared, ultrasonic, etc. Because the traditional UWB technology uses the uplink request mode [15], there is a serious delay problem when six channels are used in the system. This kind of delay problem makes the traditional UWB not suitable for the precise positioning under high-speed driving or the common precise positioning under the action of multiple vehicles.

To achieve the goal of accurate positioning in the high-speed tunnel and solve the problem of navigation and positioning in the tunnel, this paper uses the downlink time difference of arrival (DL-TDoA) technology based on UWB protocol to construct the real-time high-speed positioning system. According to the difference of signal locating time to different monitoring stations, the distance of the signal source can be determined. The advantages of the proposed DL-TDoA system contain no signal-coupling problem, low equipment complexity, and high positioning accuracy. This edge computing system shortens the response time, reduces network pressure, and provides an improved user experience $[16,17]$.

There is no phase ambiguity in the proposed DL-TDoA system, so the direction-finding baseline cannot be subject to restrictions. The traditional direction-finding method needs to calculate the azimuth angle by the phase, but the phase measurement has the uncertainty of the $2 \pi$ period. Thus, these traditional methods often use the method that the antenna baseline is smaller than the signal wavelength to avoid the $2 \pi$ period. However, the wavelength of the high-frequency signal is short, which makes the test antenna close to each other. This way, the high-frequency signal is easy to produce a signal coupling that leads to measurement error. In the proposed DL-TDoA system, each monitoring station only needs one antenna, which fundamentally solves the problem of signal coupling. In each DL-TDoA monitoring station, the system only needs to configure the monitoring antenna and receiver. The requirement of monitoring stations for the antenna is low, even if different monitoring stations use different kinds of antennas. Direction finding antenna is a group of the antenna array, where each antenna in the array is required to keep consistent. Inconsistent antenna arrangement will affect the accuracy of direction finding, which cause high system cost and interfere with monitoring performance. In the DL-TDoA monitoring station, the positioning accuracy depends on the accuracy of time measurement and vehicle speed. To verify the accuracy of DL-TDoA, this paper uses the real-time kinematic (RTK) system to measure the real vehicle trace. Through using a series of comparative experiments, the errors of vehicle speed are displayed in the experiment part. Finally, the system shows that DL-TDoA has high accuracy on the highways and tunnels and the mean errors of $11.96 \mathrm{~cm}, 37.11 \mathrm{~cm}$, $50.06 \mathrm{~cm}$, and $87.03 \mathrm{~cm}$ in the scenarios of static tests and
$30 \mathrm{~km} / \mathrm{h}, 60 \mathrm{~km} / \mathrm{h}$, and $80 \mathrm{~km} / \mathrm{h}$ in dynamic tests, respectively, which satisfy the requirements of RTLS.

Specifically, the major contributions include the following:

(1) In the proposed edge computing system, our method not only focuses on the structure of the accurate positioning system but also on the frequency and errors the system requires and the DL-TDoA brings, respectively. Based on the DL-TDoA concept, this paper provides the edge calculating equations that were used in the system and experiment

(2) Some bridge nodes, anchor nodes, and devices for highway positioning and tunnel positioning are introduced. These devices are used in commerce and gain great effects

(3) To evaluate the performance of our approach, we implement all approaches in a true ground, Xishatun test ground. The experimental results show that our approach achieves higher accuracy and efficiency as well as the baseline result of RTK

This paper is organized as follows. In Section 2, some related work is introduced. In Section 3, the proposed DLTDoA system and how to process data are described. In Section 4, the experiment devices and scenario analysis are presented. We analyze the performance of the experiment results using the proposed DL-TDoA technology and the reference technology, RTK. Section 5 concludes this paper.

\section{Related Work}

For outdoor real-time positioning, technologies, such as GPS, Beidou [18], inertial navigation, wheel ranging, and ground pseudo base station, have been developed already perfectly. This kind of technology can be combined with a real-time kinematic (RTK) system and can be applied to vehicle navigation and automatic driving in the outdoor environment. However, the research [19] shows that the above-mentioned technology does not apply to the precise positioning and navigation under the high-speed driving conditions in a closed environment such as a tunnel. The cost of some technologies such as inertial navigation is expensive, which is not suitable for the construction and implementation of large-scale projects. To solve the problem of precise positioning in this special case, an indoor real-time high-speed positioning technology is needed.

Bluetooth is a kind of radio technology that supports short-distance communication (generally within $10 \mathrm{~m}$ ) of equipment. It can exchange wireless information among many devices including mobile phones, wireless headset, and notebook computer. The transmission distance of

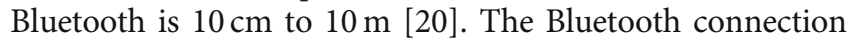
process involves multiple information transmission and verification processes, repeated data encryption, and decryption process and authentication process for each connection [21]. This process is a great waste and delay for device computing resources, which cannot meet the needs of real-time positioning. Moreover, the security of the encryption algorithm 
used in the process of Bluetooth data transmission also needs to be improved.

Radio Frequency Identification (RFID) is a kind of automatic identification technology. It can realize noncontact two-way data communication through radio frequency and read and write the recording media (electronic tag or radio frequency card) by using radiofrequency mode. RFID is used in access control, parking control, production line automation, and material management. RFID technology has some disadvantages: the technology maturity is not enough and the security is not strong enough [22]. RFID technology appeared a short time ago and is not mature in technology. The RFID tag has the characteristics of retroreflection, which makes it difficult to apply in metal, liquid, and other commodities. The security problems faced by RFID technology are mainly manifested in the illegal reading and malicious tampering of RFID electronic tag information.

ZigBee is a wireless network protocol with low speed and short-distance transmission. The main features of ZigBee are low speed, low power consumption, low cost, supporting a large number of network nodes, supporting a variety of network topologies, low complexity, fast, and reliable [23]. The combination of 802.15.4 standard makes its products have the characteristics of low power consumption, easy networking, and short distance interconnection. Therefore, its application in sensor networks or the Internet of things has considerable advantages, but its security and anti-interference performance are lower than those of similar technologies. Despite its excellent performance, ZigBee is also limited by distance.

Infrared communication technology is suitable for lowcost, cross-platform, point-to-point high-speed data connection, especially for embedded systems. Infrared transmission is wireless and cannot transmit too far. There must be no obstacles in the middle, that is to say, it cannot pass through the wall, almost unable to control the progress of information transmission.

Ultra-wideband (UWB) is a new type of wireless communication technology, initially used for military purposes. UWB mainly uses a very short pulse signal to transmit data, which can ensure high-speed communication, but the transmission power is very small [24]. UWB does not need to use the carrier of the traditional communication system but transmits and receives very narrow pulses with nanosecond or below to transmit data, so it has the bandwidth of $\mathrm{GHz}$ order. The main advantages of UWB include low power consumption, insensitive to channel fading, strong anti-interference ability, no interference to other equipment in the same environment, strong penetration (positioning in the environment penetrating a brick wall), high security, low system complexity, and accurate positioning. Therefore, UWB technology can be applied to indoor static or moving objects and human positioning, tracking, and navigation and can provide very accurate positioning accuracy.

Download time difference of arrival (DL-TDoA) is a new patented technology of downlink broadcast ultra-wideband. It is a method of positioning by using time difference [25]. The distance of the signal source can be determined by measuring the time when the signal arrives at the monitoring sta- tion. The advantage of DL-TDoA is that there is no phase ambiguity, so the direction-finding baseline can be unlimited. DL-TDoA has the characteristics of low complexity, for TDoA monitoring station only needs to configure the monitoring antenna and receiver, and the requirements for the antenna are not high, even if different monitoring points use different antennas. The positioning accuracy of the TDoA detection station depends on the accuracy of time measurement. Through the optimized algorithm, the calculation error of time difference is in the order of $100 \mathrm{~ns}$. Comparing with the other positioning algorithms, like MDS-MAP [26], DL-TDoA outperforms at high-speed calculating.

From the aspect of positioning technology, no matter the positioning accuracy, security, anti-interference, power consumption, etc., the combination technology based on Beidou, DL-TDoA, and visual positioning is one of the most ideal precision positioning and navigation technologies under high-speed driving.

To verify the accuracy of DL-TDoA, an ultrahigh-precision positioning technology is needed for comparison. RTK is a commonly used high-precision GPS measurement and positioning technology. RTK can obtain centimeter-level ultraprecision outdoor positioning results [27]. Through the carrier phase dynamic real-time difference method, the accuracy of positioning and measurement is greatly improved. The RTK method is less affected and limited by visibility, climate, season, and other factors and can implement high-precision positioning in complex terrain areas [28]. Therefore, this paper uses RTK as a comparative reference technology.

\section{Automated RTLS Using DL- TDoA Technology}

This section describes the proposed automated location solution using a DL-TDoA technology for precise vehicle positioning under highway and tunnel scenarios. The designed architecture of the system is briefly introduced in Sections 3.1-3.3. Section 3.4 introduces the scenario constructions of highway and tunnel. Section 3.5 describes the hardware devices such as tags, anchors, and applications in detail.

\subsection{The Comparison with DL-TDoA and Traditional UWB.} The main advantages of traditional UWB are low power consumption, insensitive to channel fading, strong antiinterference ability, no interference to other equipment in the same environment, especially when facing lots of vehicles on channel highways, strong penetration (positioning in the environment penetrating a brick wall), and high positioning accuracy. DL-TDoA inherits the excellent features of UWB and develops its new features of real-time positioning. As shown in Figure 1, tradition UBW utilizes the time difference algorithm to calculate the distances between the vehicle and bridge nodes. The UWB router transfers the time differences to the location computing server to calculate the position. DL-TDoA relies on edge computing to reduce the server calculating pressure. In the DL-TDoA framework, the sensor owns edge computing ability to calculate the distances between anchor nodes and the positioning sensor. The sensor transmits the position data to the bridge node with a $4 \mathrm{~Hz}$ 

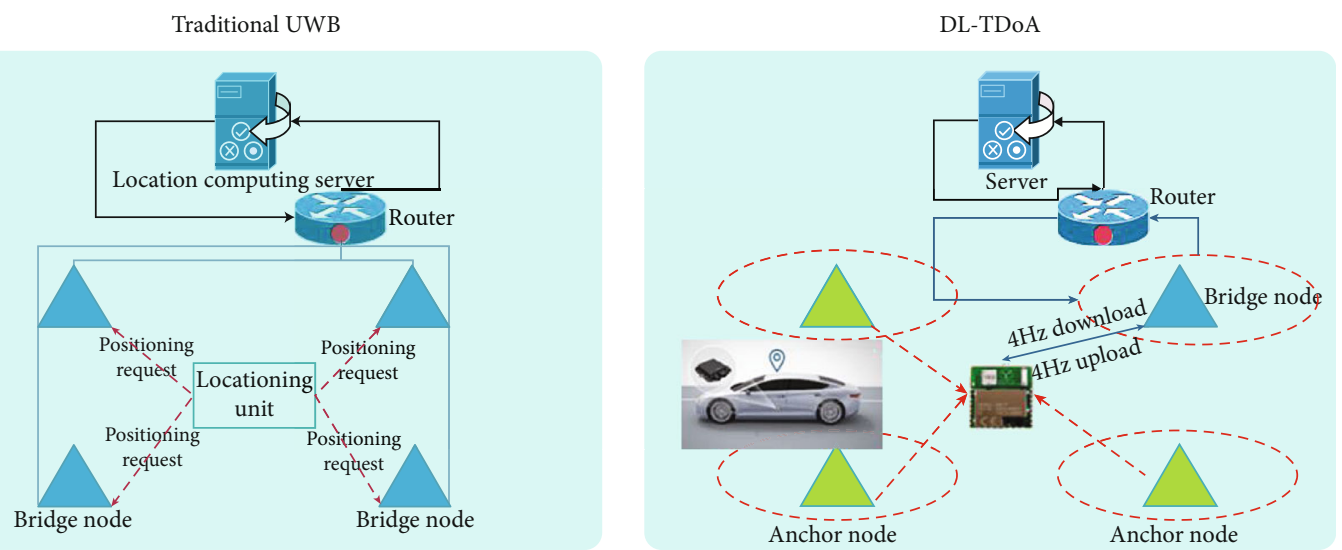

Figure 1: The difference between DL-TDoA and UWB.

upload and download frequency. Then, the router and server just transfer and save the date and the server avoids computing any positioning information. In DL-TDoA, the computing work is assigned to every vehicle to calculate its position, in which way, only one bridge node uploads data while traditional UWB has more than one bridge node. Anchor nodes use the broadcasting protocol, which avoids the time of network verification and save the fee of the public network. Highway and tunnel positioning systems require real-time data so DL-TDoA is better than the traditional UBW to satisfy the real-time requirement and provides a smart mesh network to reduce the computing for the server.

In the traditional UWB system, the data from the positioning tag or sensor is calculated by the server so there is some uploading time. When the car opens the navigation system in the tunnel, the positioning tag has to request the position data from the server so there is some downloading time. The delay of upload and download prevents the traditional UWB system from becoming a real-time positioning system. However, the proposed DL-TDoA uses the edge computing mechanism to obtain a real-time position without any more delay. Moreover, DL-TDoA also uploads the position data to the server for mobile monitoring and analysis. DL-TDoA utilizes the frequency of $1 / 2 / 4 \mathrm{~Hz}$ to upload, which can be configured by needs, while the USB frequency for DLTDoA edge computing and navigation can reach $26 \mathrm{~Hz}$ without uploading. Traditional UWB cannot serve the navigation applications. Due to the edge computing and the release of server consumption in DL-TDoA, the capacity of the anchor nodes and bridge nodes has no limitations while that of traditional UWB is usually less than 300 vehicles. DL-TDoA uses the anchor nodes and bridge nodes to form the ad hoc network, which utilizes the local network and requires a low price compared with the station using the public network. Therefore, the real-time and low price DL-TDoA solves the problems that the highway and tunnel real-time positioning bring.

3.2. Automated Highway RTLS Overview by Using DL-TDoA. As described in Figure 2, the proposed automated location solution mainly contains 6 groups of devices, including various tags, anchor or bridge nodes, exchangers, routers, networks, and RTLS server. Several common tags are listed in the bottom part of the figure, such as sensor tag, badge tag, asset tag, and a positioning unit. These tags are connected to the wireless infrastructure network (IPV6 mesh), which consists of massive anchor and bridge nodes. The data collected by tags are uploaded to the exchanger through the infrastructure network. When these data continue uploading from the exchanger to the router, their transmission range is converted from a personal domain network to a public domain network. After these data arrive at the RTLS through the network, a series of applications, software, information broad, report, and network services use these data to display locations and analyze results.

According to the mathematical principle, the locus of a moving point whose distance difference from two fixed points is constant is a hyperbola. To determine a point in three-dimensional space, at least three distance differences and four observation points are needed. Therefore, there should be at least four observation stations for positioning.

In the DL-TDoA edge computing tag, the practicability of the localization algorithm affects the result of localization. Assume that the coordinate of the target, a vehicle, is $(x, y, z)$. Now, the DL-TDoA system has at least $M+1$ anchor nodes, and one of these nodes is the main station $S_{0}$ while the others are the substation $S_{i}$, and the coordinates are $\left(x_{i}, y_{i}, z_{i}\right), i=0,1,2,3, \cdots, M$. Suppose that the time of electromagnetic radiation from the target to each station is $t_{i}, i=0,1,2,3 \cdots M$. The time difference between the arrival times of each substation and that of the main station can be written as $\pi_{i}, i=1,2,3, \cdots, M$. By multiplying the time difference of arrival by the speed of light, the distance difference between the vehicle and each substation to the terminal can be obtained:

$$
\Delta r_{i}=c \pi_{i}
$$

where $c$ represents the theoretical speed of light. The distance difference can also be obtained directly from the 


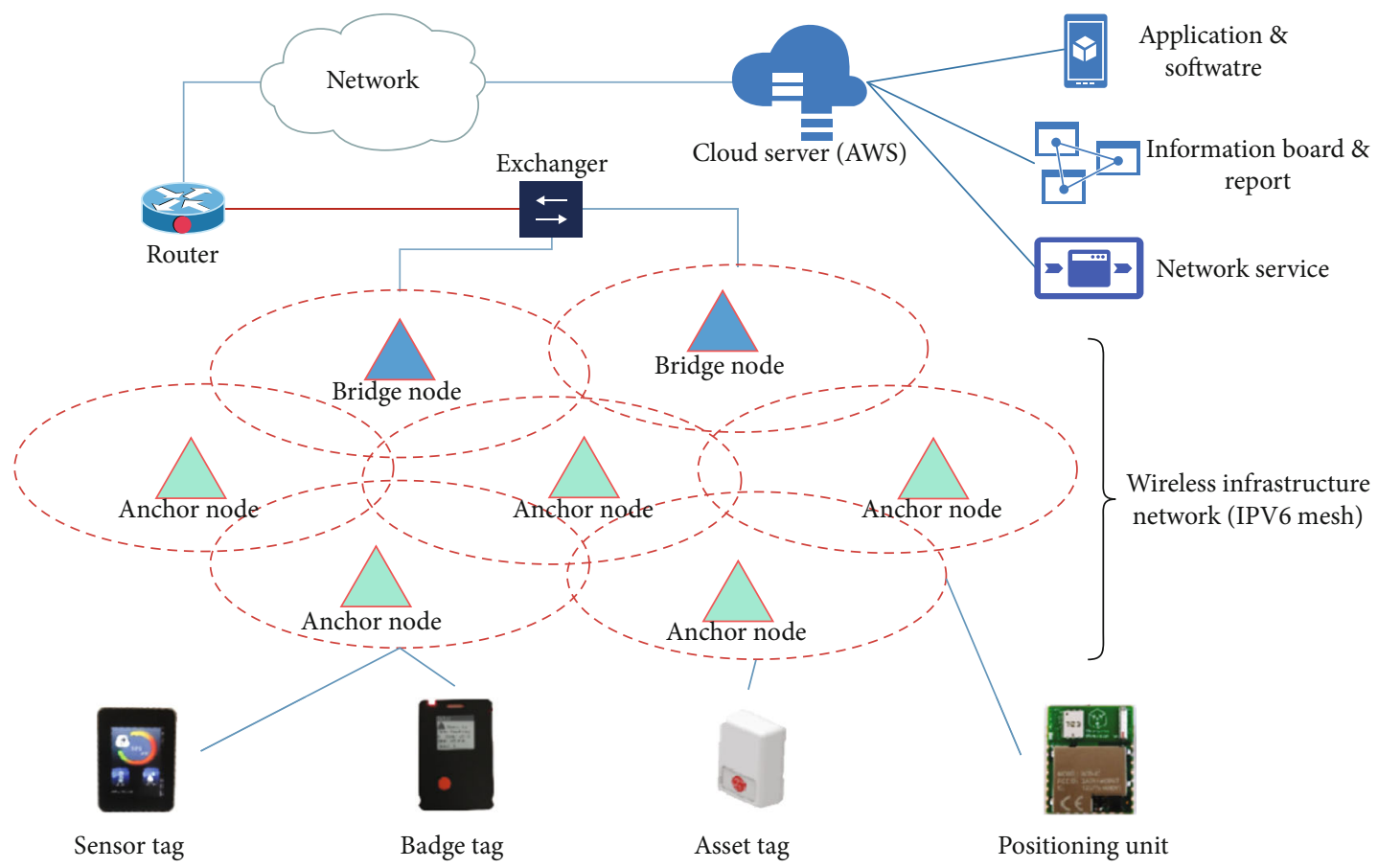

FIGURE 2: The architecture of the proposed automated location solution using DL-TDoA.

distance between the target and the main station minus the distance between the target and the substation:

$$
\begin{aligned}
\Delta r_{i}= & r_{i}-r_{0}=\sqrt{\left(x-x_{i}\right)^{2}+\left(y-y_{i}\right)^{2}+\left(z-z_{i}\right)^{2}} \\
& -\sqrt{\left(x-x_{0}\right)^{2}+\left(y-y_{0}\right)^{2}+\left(z-z_{0}\right)^{2}} .
\end{aligned}
$$

In equation (2), the part of $\sqrt{\left(x-x_{0}\right)^{2}+\left(y-y_{0}\right)^{2}+\left(z-z_{0}\right)^{2}}$ equals $r_{0}$; the next equation is obtained:

$$
\left(\Delta r_{i}+r_{0}\right)^{2}=r_{i}^{2}=\left(x-x_{i}\right)^{2}+\left(y-y_{\mathrm{i}}\right)^{2}+\left(z-z_{i}\right)^{2} .
$$

Since $r_{0}$ is an unknown parameter, the inequality is reduced to a linear equation and $r_{0}$ is eliminated. Subtract $r_{0}^{2}$ from both sides of the formula:

$$
\begin{aligned}
\Delta r_{i}^{2}+2 \Delta r_{i} r_{0}= & 2\left[x\left(x_{0}-x_{i}\right)+y\left(y_{0}-y_{i}\right)+z\left(z_{0}-z_{i}\right)\right] \\
& +\left(x_{i}^{2}+y_{i}^{2}+z_{i}^{2}\right)-\left(x_{0}^{2}+y_{0}^{2}+z_{0}^{2}\right) .
\end{aligned}
$$

Let $d_{i}$ represent the distance of each station, $d_{i}^{2}=\left(x_{i}^{2}+\right.$ $\left.y_{i}^{2}+z_{i}^{2}\right)$, then

$$
\Delta r_{0}^{2}+2 \Delta r_{i} r_{0}=2\left[x\left(x_{0}-x_{i}\right)+y\left(y_{0}-y_{i}\right)+z\left(z_{0}-z_{i}\right)\right]+d_{i}^{2}-d_{0}^{2} .
$$

It can be sorted out:

$$
\Delta r_{i} r_{0}+\frac{\Delta r_{i}^{2}-d_{i}^{2}+d_{0}^{2}}{2}=x\left(x_{0}-x_{i}\right)+y\left(y_{0}-y_{i}\right)+z\left(z_{0}-z_{i}\right)
$$

where $i=1,2,3, \cdots, m$ of the above equation represents the number of anchor nodes and $x, y, z$ are the unknown numbers, so the above equation is rewritten as the following matrix:

$$
\begin{gathered}
{\left[\begin{array}{ccc}
x_{0}-x_{1} & y_{0}-y_{1} & z_{0}-z_{1} \\
\vdots & \vdots & \vdots \\
x_{0}-x_{m} & y_{0}-y_{m} & z_{0}-z_{m}
\end{array}\right]\left[\begin{array}{c}
x \\
y \\
z
\end{array}\right]} \\
=\left[\begin{array}{c}
\Delta r_{1} \\
\vdots \\
\Delta r_{m}
\end{array}\right] r_{0}+\left[\begin{array}{c}
\frac{\Delta r_{1}^{2}-d_{1}^{2}+d_{0}^{2}}{2} \\
\vdots \\
\frac{\Delta r_{i}^{2}-d_{i}^{2}+d_{0}^{2}}{2}
\end{array}\right] .
\end{gathered}
$$

The matrix of formula (7) can be divided into

$$
A=\left[\begin{array}{ccc}
x_{0}-x_{1} & y_{0}-y_{1} & z_{0}-z_{1} \\
\vdots & \vdots & \vdots \\
x_{0}-x_{m} & y_{0}-y_{m} & z_{0}-z_{m}
\end{array}\right],
$$




$$
B=C r_{0}+D=\left[\begin{array}{c}
\Delta r_{1} \\
\vdots \\
\Delta r_{m}
\end{array}\right] r_{0}+\left[\begin{array}{c}
\frac{\Delta r_{1}^{2}-d_{1}^{2}+d_{0}^{2}}{2} \\
\vdots \\
\frac{\Delta r_{i}^{2}-d_{i}^{2}+d_{0}^{2}}{2}
\end{array}\right] .
$$

According to the linear properties of linear equations, the solution set of $A X=B$ is the sum of the solution sets of $A X=C r_{0}$ and $A X=D$. When $m=3, A$ is a square matrix. According to the Cramer rule, its solution can be expressed as

$$
x_{i j}=\frac{\left|A_{j}\right|}{|A|},
$$

where $A_{j}$ is the determinant obtained by replacing the $j$ th column element in $a$ with a constant term. The new equation can be obtained:

$$
\left\{\begin{array}{l}
x=a_{1} r_{0}+b_{1}, \\
y=a_{2} r_{0}+b_{2}, \\
z=a_{3} r_{0}+b_{3},
\end{array}\right.
$$

where $a_{i}$ is the solution set of $A X=C$ and $b_{i}$ is the solution set of $A X=D$. Then, replace the $x, y, z$ variables of equation (12) with those in equation (11):

$$
\begin{aligned}
r_{0}^{2}= & \left(x-x_{0}\right)^{2}+\left(y-y_{0}\right)^{2}+\left(z-z_{0}\right)^{2}, \\
r_{0}^{2}= & \left(a_{1}^{2}+a_{2}^{2}+a_{3}^{2}\right) r_{0}^{2}+2 r_{0}\left(a_{1} b_{1}+a_{2} b_{2}+a_{3} b_{3}-a_{1} x_{0}-a_{2} y_{0}-a_{3} z_{0}\right) \\
& +\left(x_{0}-b_{1}\right)^{2}+\left(y_{0}-b_{2}\right)^{2}+\left(z_{0}-b_{3}\right)^{2} .
\end{aligned}
$$

Suppose $\alpha=\left(a_{1}^{2}+a_{2}^{2}+a_{3}^{2}\right), \quad \beta=\left(a_{1} b_{1}+a_{2} b_{2}+a_{3} b_{3}-a_{1}\right.$ $\left.x_{0}-a_{2} y_{0}-a_{3} z_{0}\right)$, and $\gamma=\left(x_{0}-b_{1}\right)^{2}+\left(y_{0}-b_{2}\right)^{2}+\left(z_{0}-b_{3}\right)^{2}$ , then the final equation is

$$
r_{0}=\frac{-\beta \pm \sqrt{\beta^{2}-4 \alpha \gamma}}{2 \alpha} .
$$

When $r_{0}$ has two solutions, two positioning results are obtained, that is, the positioning is fuzzy, so the observation station needs to be added. When $r_{0}$ has a solution, the target position can be uniquely determined. When $r_{0}$ has no solution, the position cannot be determined. After $r_{0}$ is obtained, $x, y, z$ can be found in equation (11). Through the edge calculation of the positioning tag on the vehicle, the position information returned by the DL-TDoA algorithm is calculated in the tag to carry out accurate positioning processing.

3.3. RTK and DL-TDoA Data Format Processing. RTK positioning system (high-precision GPS measurement and positioning technology), as a comparative technology, DLTDoA data should be transformed into RTK format in the same coordinate. The original longitude and latitude of
RTK data are transformed as international format: degrees, minutes, and seconds. Since the original RTK data collection is based on the geodetic coordinate system, the WGS-84 ellipsoid datum algorithm transforms the coordinate system of the data from the geodetic coordinate system to a $x, y, z$ plane coordinate system. In this case, the origin of the coordinate system is not the origin specified by the test site, so it is necessary to translate the data to the coordinate system centered on the specified origin. The data on the other side is DL-TDoA data. The data of DL-TDoA is the data of the plane coordinate system, but the data needs to be rotated to the same $x, y, z$ direction the same as RTK data. Since the uplink DL-TDoA frequency is $4 \mathrm{~Hz}$, while the RTK upload frequency is $26 \mathrm{~Hz}$, it is necessary to use the difference compensation method to find the missing values, to achieve the same frequency as RTK. As the RTK and DL-TDoA antenna cannot be fixed at the same position on the vehicle, there is a physical error to compare the traces. Therefore, the data from DL-TDoA should manually minus the error distance. Now, the RTK and DL-TDoA data are in the same coordinate, and no outside errors do not come from algorithms.

3.4. Highway and Tunnel Scenario Setup. As shown in Figure 3(a), the highway simulation environment is selected in the Xishatun test ground. The green area is the test area, and the two ends are the start point and the endpoint, respectively. At this time, the area is a straight barrier-free road, and there is no signal occlusion. The test area is described (Figure 3(b)). First of all, the acceleration part and deceleration part of the vehicle are green areas, and the green area is not included in the data analysis plan, because the main test of DL-TDoA is still focused on driving at a constant speed. The total length of the test area is $130-150 \mathrm{~m}$, and the length of the DL-TDoA positioning test area is $100 \mathrm{~m}$. Nodes are set in the gantry area as shown in the test (Figure 3(c)). Due to the natural conditions of the test area, the distance between the gantries is not the same. The interval is about $8 \mathrm{~m}$ or $30 \mathrm{~m}$. To increase the positioning effect, the test area requires some artificial anchor points, which may not be fixed on the gantry because the number of gantries is fixed. Therefore, temporary anchor points for tripod support are added on both sides of the road in Figure 3(d) (green anchor points are temporary anchor points while red ones are fixed anchor points fixed on gantry). Anchors 7 and 8 are bridge nodes, which link to the public network to upload the positioning track. The accuracy of DL-TDoA on the highway is verified to determine whether it can perform well in the tunnel.

The arrangement of anchor nodes and bridge nodes on both sides of the tunnel is shown in Figure 4. Because there are rocks and walls on both sides of the tunnel, it is more effective to arrange joints on the wall. Anchor nodes and bridge nodes are arranged alternately to obtain a better ad hoc network.

3.5. Anchor and Tag Devices. To solve the time-consuming and labor-consuming problem of coordinate measurement in the anchor node, the self-positioning module of the anchor node is developed in this paper. After the installation of the 


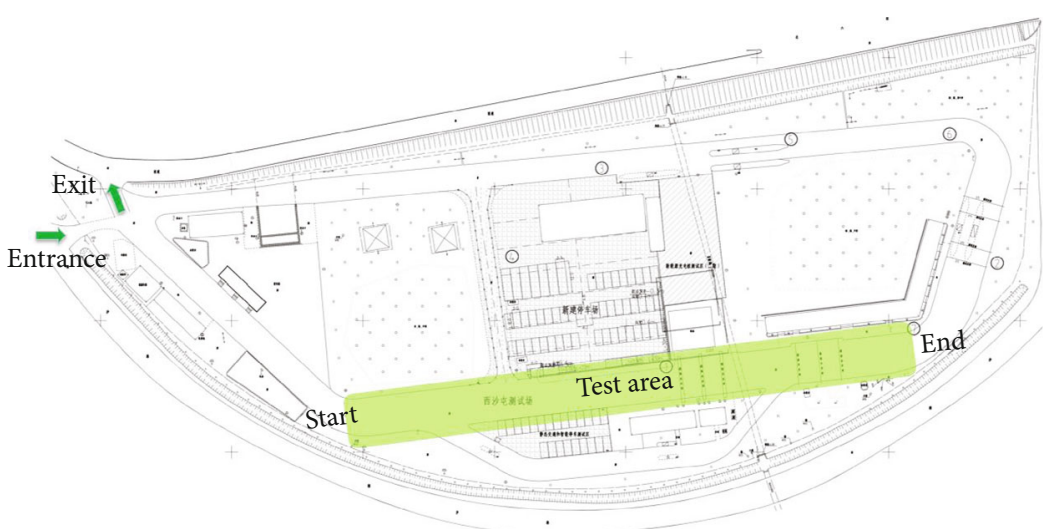

(a)

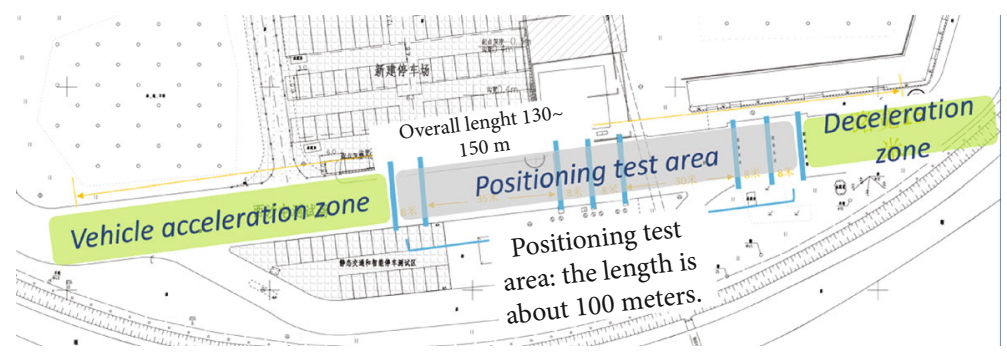

(b)

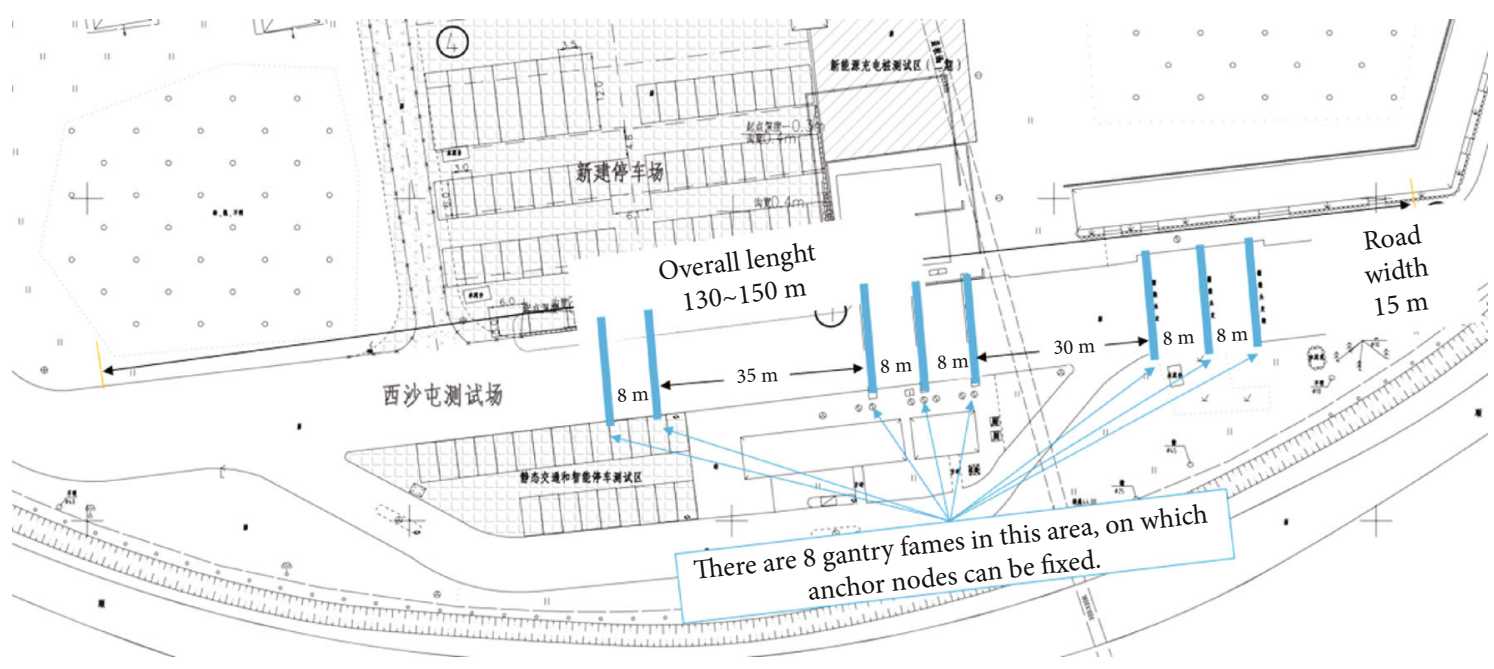

(c)

Figure 3: Continued. 


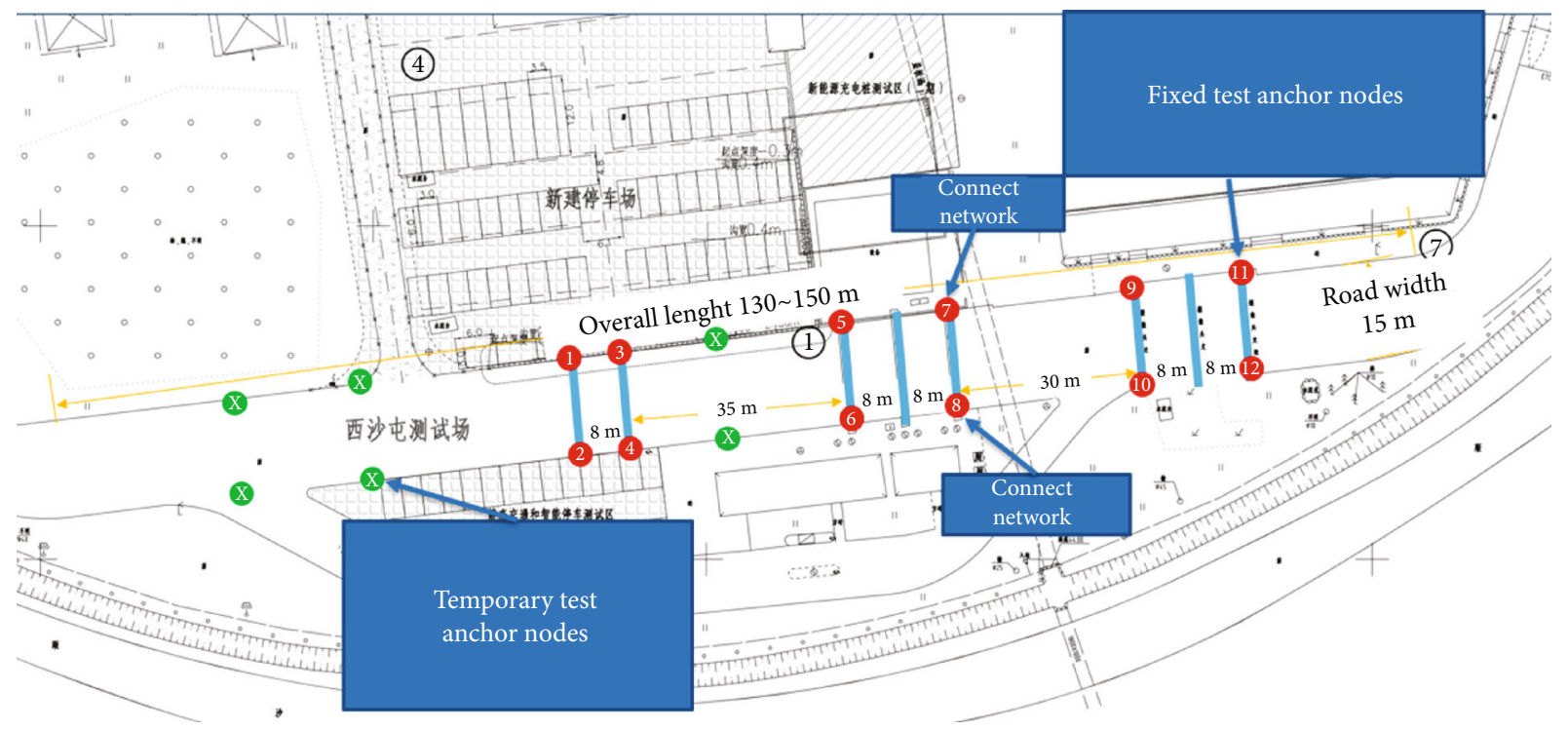

(d)

Figure 3: Anchor and bridge node deployment for the DL-TDoA highway test. (a) Test area: Xishatun test ground. (b) The test area includes three parts: the acceleration zone, the test area, and the deceleration zone. (c) Gantry distances. (d) The distribution of anchor and bridge nodes.

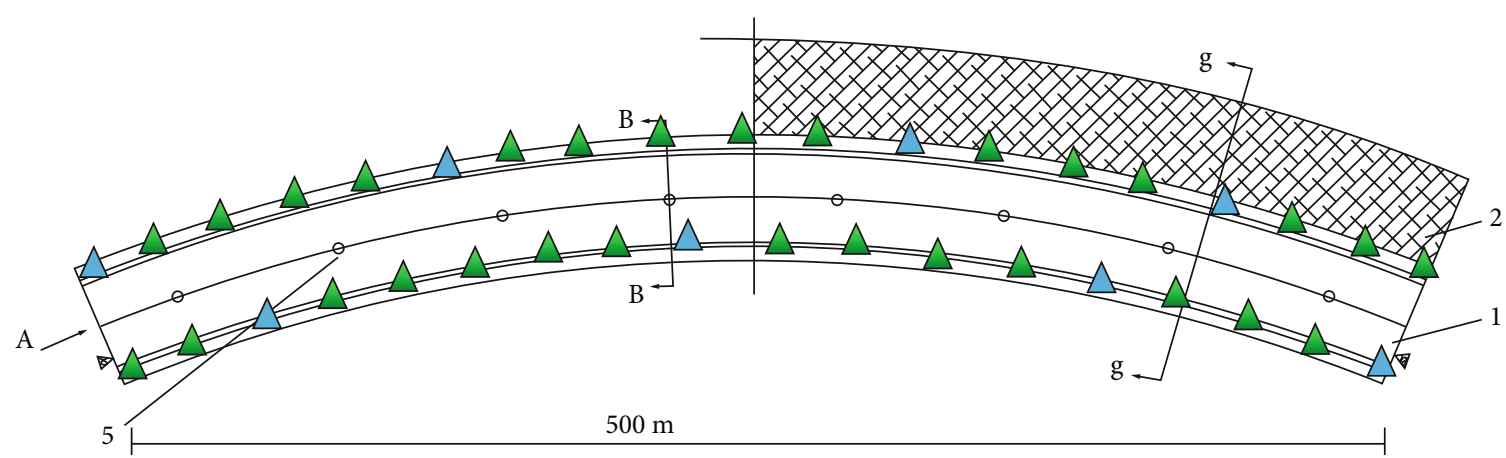

$\triangle$ Bridge node

$\triangle$ Anchor node

FIGURE 4: The distribution of anchor and bridge nodes in the tunnel test.

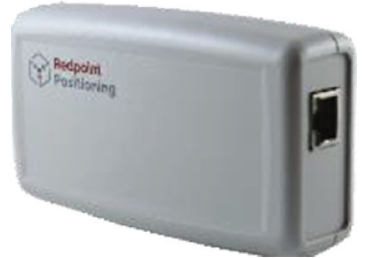

(a)

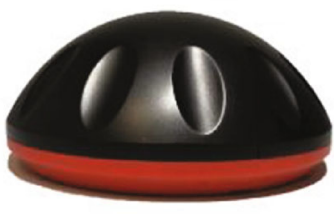

(b)

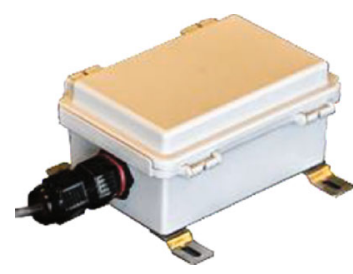

(c)

FIGURE 5: Three common anchor node devices adopted in the proposed solution. (a) Wall-mounted anchor node. (b) Roof anchor node. (b) Three-defense anchor node.

equipment, the system can automatically measure the coordinates of anchor nodes, which establish a coordinate system and simplify the construction process. Figure 5 displays three common anchor node devices, including wall-mounted anchor nodes, roof anchor nodes, and three defense anchored nodes. The wall-mounted anchor node in Figure 5(a) can be hanging fixed on the vertical wall. The roof anchor node in Figure 5(b) is always fixed with clamps or screws on indoor ceilings. The three-defense anchor node in Figure 5(c) adopts a screw fixation method that can be installed in both indoor and outdoor environments. All three kinds of anchor nodes are powered by PoE supply. 


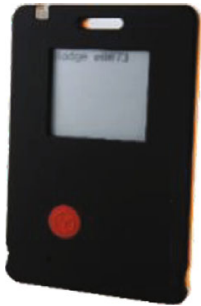

(a)

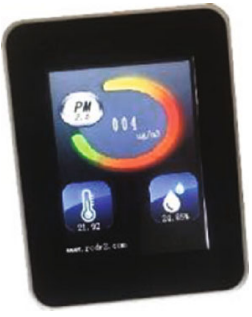

(d)

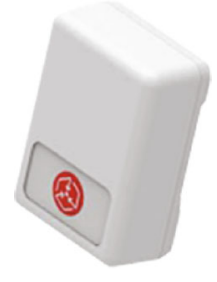

(b)

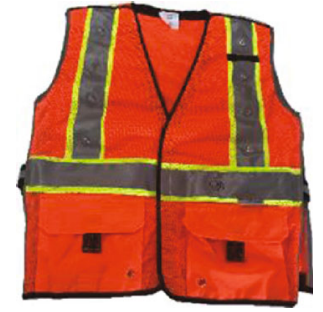

(e)

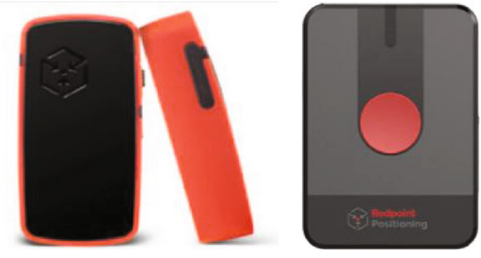

(c)

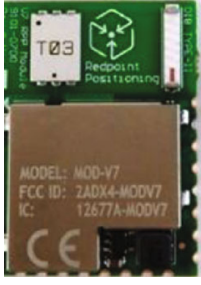

(f)

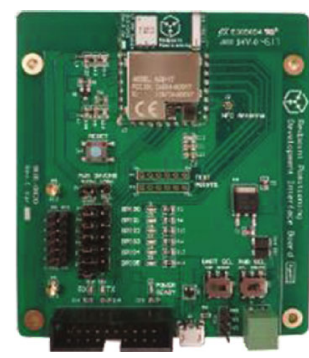

(g)

Figure 6: Seven common tags and units adopted in the proposed solution. (a) Badge tag. (b) Asset tag. (c) General tag. (d) Sensor tag. (e) Safety vest. (f) Positioning unit. (g) V7 development board.

In the proposed solution, the wireless network connects the anchor nodes, where each anchor node only needs PoE power. About $15 \%$ of anchor nodes in the network need to be connected to the switch. In the construction process of the proposed solution, all wireless networks make the deployment of anchor nodes easier and the construction cost is greatly reduced. Based on the calculation of the same number of base stations, the cost of construction and auxiliary materials of our solution will be saved by about $70 \%$. Moreover, because most anchor nodes need power supply tightly, they will be more flexible in the location selection of base stations.

The DL-TDoA algorithm proposed in this paper can use the tag to calculate the location information, making the capacity of the network theoretically unlimited. In the actual application scenario, it is enough to support a large number of tags. Based on wireless communication technology, anchor nodes automatically form an IPv6 mesh network, which has very flexible scalability. If there exists a deployed base station under the environment, the newly installed base station will automatically join the existing mesh network if the location area needs to be increased.

Figure 6 displays seven common tags and positioning units, including badge tag, asset tag, general tag, sensor tag, safety vest, positioning unit, and V7 development board. In Figure 6(a), the badge tag is always used for tracking personnel. The device owns one button with an alarm function. When the alarm is triggered, the device makes a sound, its LED stroboscopic alarm, and its electronic screen display. The device is wearable and with a single rechargeable lithium battery. Figure 6(b) displays an asset tag that is always used to track assets or objects with low moving frequency. The device can be fixed on objects, which also contains one alarm button and an LED stroboscopic alarm. The general tag in Figure 6(c) is always used to track vehicles or people, which can be fixed to objects or carried by peo-
TABLE 1: Device list used in test.

\begin{tabular}{lccccc}
\hline No. & Device name & Count & No. & Device name & Count \\
\hline 1 & Bridge node & 2 & 7 & Router & 1 \\
2 & Anchor node & 16 & 8 & Distance meter & 2 \\
3 & Location server & 1 & 9 & Total station & 1 \\
4 & PoE exchanger & 1 & 10 & Laptop & 2 \\
5 & Network cable & $200 \mathrm{~m}$ & 11 & PoE extender & 2 \\
6 & V7 general tag & 4 & & & \\
\hline
\end{tabular}

ple. The device owns an LED stroboscopic alarm with a sensor interface. The sensor tag in Figure 6(d) integrates a positioning module that can collect and upload sensor data with time and space labels. The device supports integration in the third party to design personalized products. The safety vest as shown in Figure 6(e) owns a wearable positioning label with light prompt and alarm function. It is suitable for the production environment of various industries: lithium battery (rechargeable) power supply. The positioning unit in Figure 6(f) contains complete core positioning functions, the firmware is preloaded, and the development of various application interfaces is supported. Support the design of personalized labels or other products on the third-party PCB. The PCB of the V7 development board in Figure 6(g) integrates a positioning module that provides multiple interfaces for the third party. Its external power supply is required as no more than $3.6 \mathrm{~V}$ through the USB power supply.

Each tag has its computing power and can calculate the location information independently. Considering the realtime requirement in RTLS, our tags can compute the location information by themselves, to achieve almost zero delays. For scenes with high real-time requirements, such as anticollision and unmanned driving, the proposed system has great advantages. The proposed solution has realized the active control of 


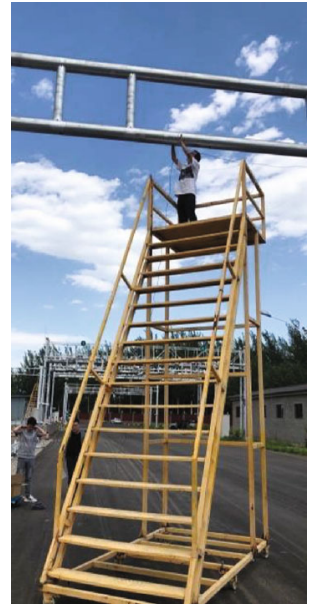

(a)

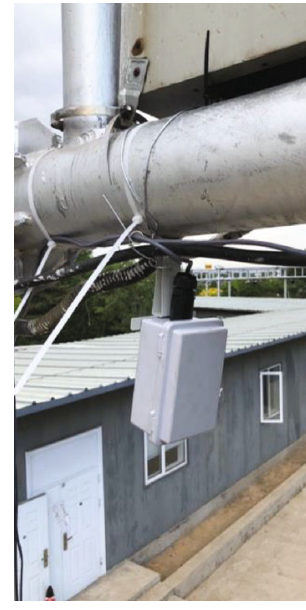

(b)

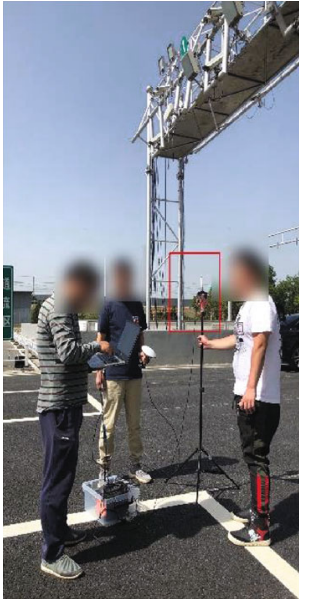

(c)

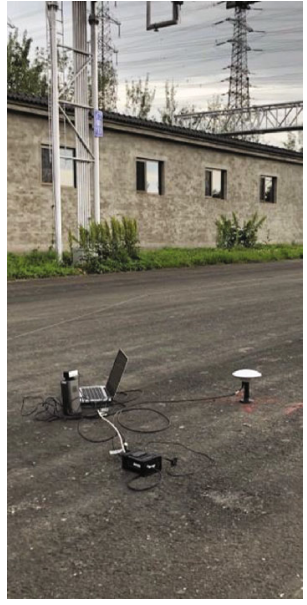

(d)

FIgURE 7: Scenario was set up. (a) Installing anchor nodes. (b) Fixed nodes. (c) Testing in different positions. (d) Collecting data.
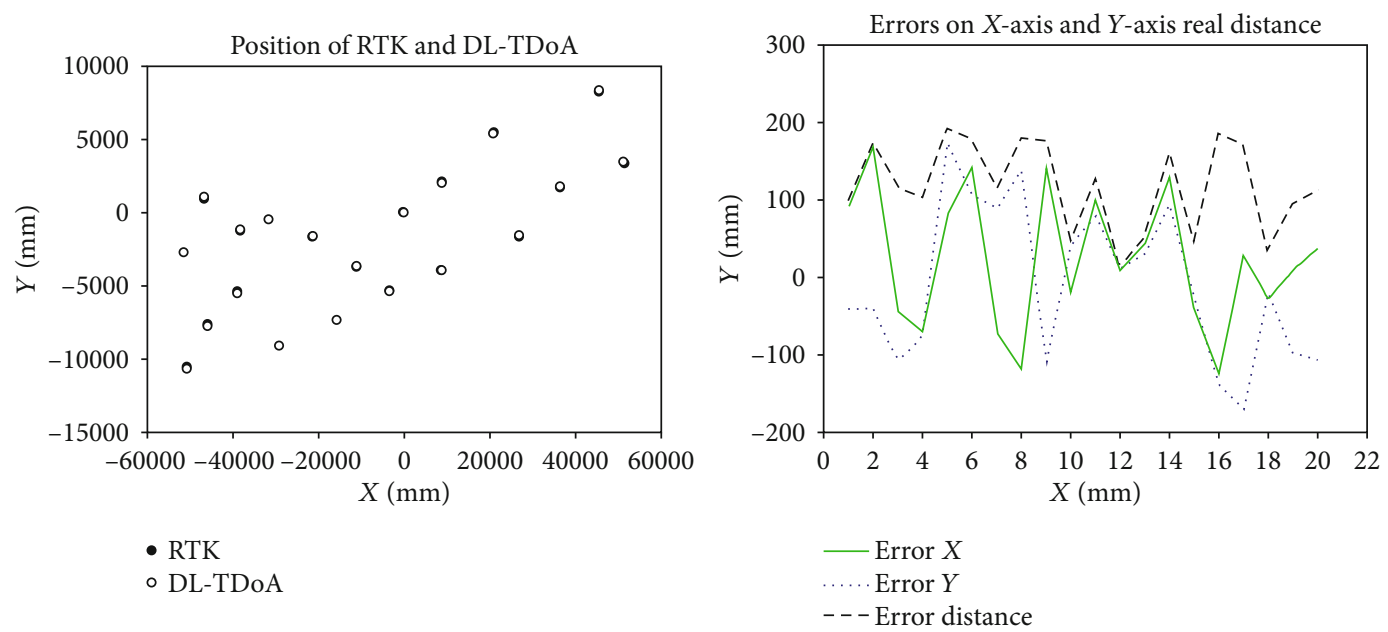

Figure 8: Static DL-TDoA and RTK test comparison.

a forklift and other machinery in the storage field and has made a lot of technical reserves for unmanned driving.

\section{Experiments and Scenario Analysis}

The verification experiment of this paper is divided into two application scenarios: highway and tunnel internal test. The devices used in the experiment are listed in Table 1. We use 2 bridge nodes, 16 anchor nodes, 1 location server, 1 PoE exchanger, $4 \mathrm{~V} 7$ general tags, 1 router, 2 distance meters, 1 total station, 2 laptops, and $2 \mathrm{PoE}$ extenders. Moreover, a $200 \mathrm{~m}$ network cable is used in the experiment.

4.1. Scenario 1: Highway Static Test. Before the test, the devices and sensors are fixed on gantries and connected to the IPV6 network and local network. As shown in Figures $7(a)$ and $7(b)$, the anchor and bridge nodes are fixed on gantries. To verify that the proposed DL-TDoA is accurate enough, first of all, the static position comparative experiments are needed. Testers prepare a triangle bracket (Figure $7(\mathrm{c})$ ), where the RTK positioning sensor and DL-
TDoA positioning sensor are alternated. The fixed position of the triangle bracket guarantees the same position for both two positioning sensors so there are no sensor physical position errors. Testers choose several positions to collect the position data for future comparisons. Figure 7 (d) shows that the positioning sensor connects the laptop directly in the static test scenario. Static test data is collected by local USB transmission.

The RTK data obtained after the fixed-point test will be converted into a plane coordinate system (using WGS-84 ellipsoid datum). It is easy to transform the RTK from the origin of the plane coordinate system to the coordinate system with the fixed point of the test field as the origin. For the data of DL-TDoA, the plane coordinate system is used as the north direction of the $y$-axis, and the original coordinate system adopted by DL-TDoA is rotated. Because the sensor positions of RTK and DL-TDoA are the same, the position difference between RTK and DL-TDoA antenna is removed and the coordinates are corrected. Finally, the distance difference between RTK and DL-TDoA is calculated point to point, and the average value of the distance difference is calculated 


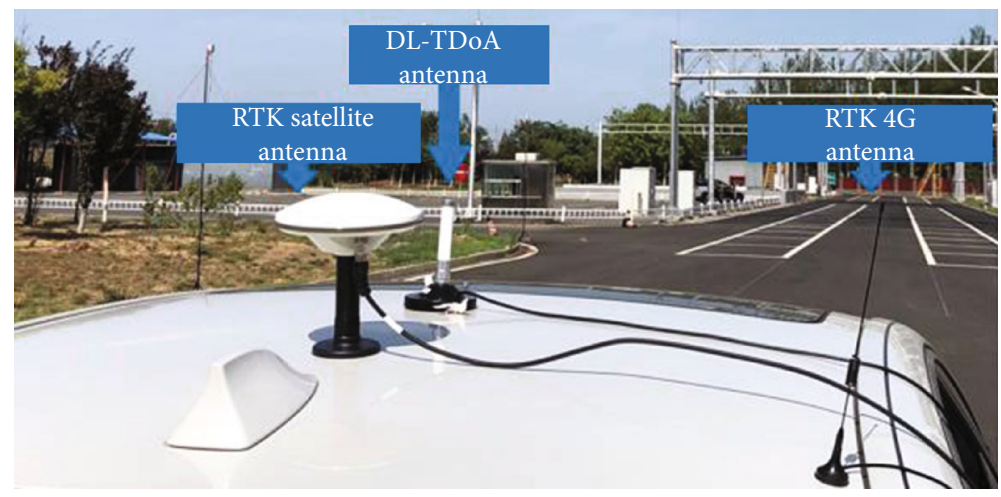

(a)

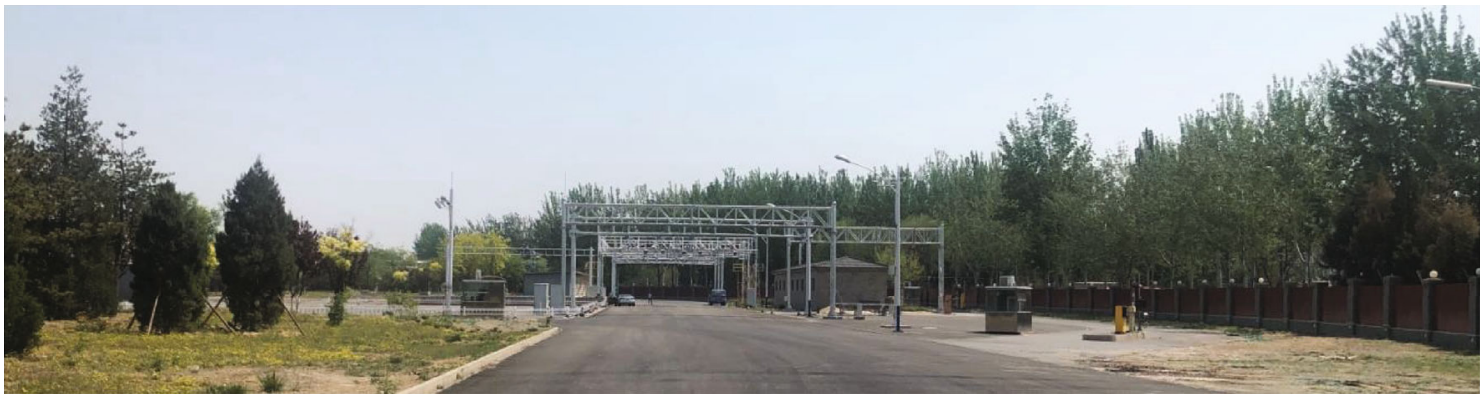

(b)

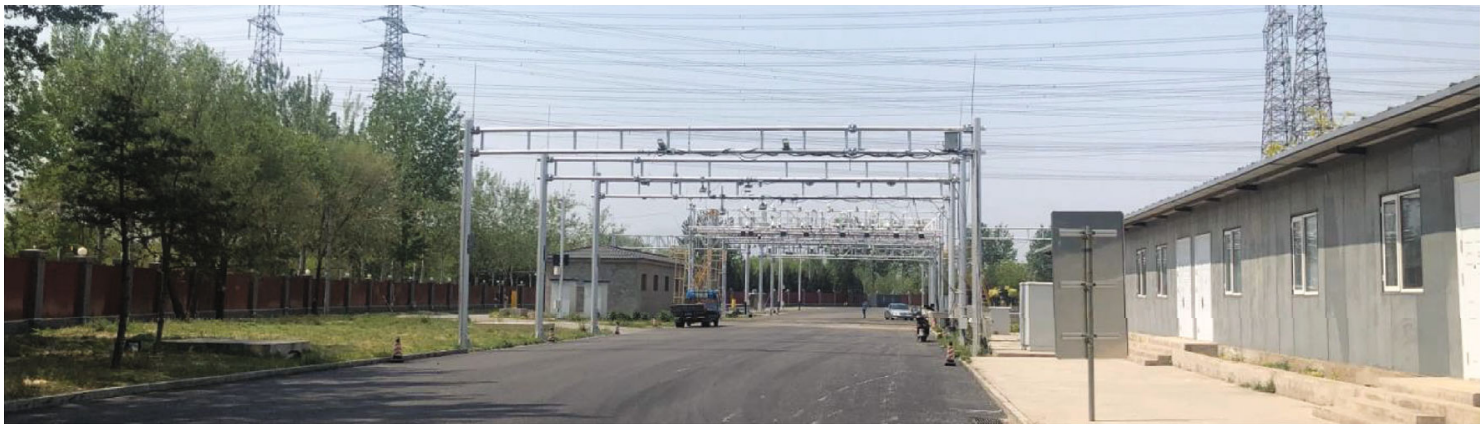

(c)

Figure 9: Dynamic scenario was set up.

to get the final result. Figure 8(a) shows the RTK and DLTDoA data in the same rectangular coordinate system. Black points represent RTK while the white points present the DLTDoA data. As shown in the figure, the majority of the tested positions is overlapped and provides excellent accuracy. For some point, only the DL-TDoA data can be seen, which proves RTK and DL-TDoA data is full the same under the millimeter unit. Figure 8(b) shows the error under static positioning. It is seen from Figure 8(b) that the maximum error is less than $200 \mathrm{~mm}$ and the minimum error is close to $0 \mathrm{~mm}$. The maximum distance error is $193.12 \mathrm{~mm}$, and the minimum distance error is $14.43 \mathrm{~mm}$. The average error of RTK and DL-TDoA was $119.64 \mathrm{~mm}$.

4.2. Scenario 1: Highway Dynamic Test. For the dynamic test, RTK and DL-TDoA sensors are fixed on the top of the vehicle. As shown in Figure 9(a), there is a $20 \mathrm{~cm}$ distance between two kinds of antennas, which causes the deviation when tracing the trajectory of two motions. Therefore, comparing with the static test, when processing the data, testers are required to manually modify the deviation, by simply subtracting $20 \mathrm{~cm}$. Another problem is that RTK transmits the data with a $26 \mathrm{~Hz}$ frequency while the frequency of DLTDoA is only $4 \mathrm{~Hz}$. Tester manually finds the same position in the same frame. These two problems are the main differences between the static and dynamic tests. Figures 9(b) and 9(c) are the start and end positions for the dynamic test. Then, the vehicle goes through the gantries, which are fixed with DL-TDoA anchor nodes and bridge nodes. The overall distance of the dynamic test is $100 \mathrm{~m}$.

In the dynamic test, three comparative experiments are set to speeds of 30,60 , and $80 \mathrm{~km} / \mathrm{h}$, respectively. For the dynamic data format, due to the deviation between RTK and DL-TDoA antenna, testers are supposed to minus the distance of that error on the coordinate. Another problem this paper has mentioned is that the uploading frequency of 

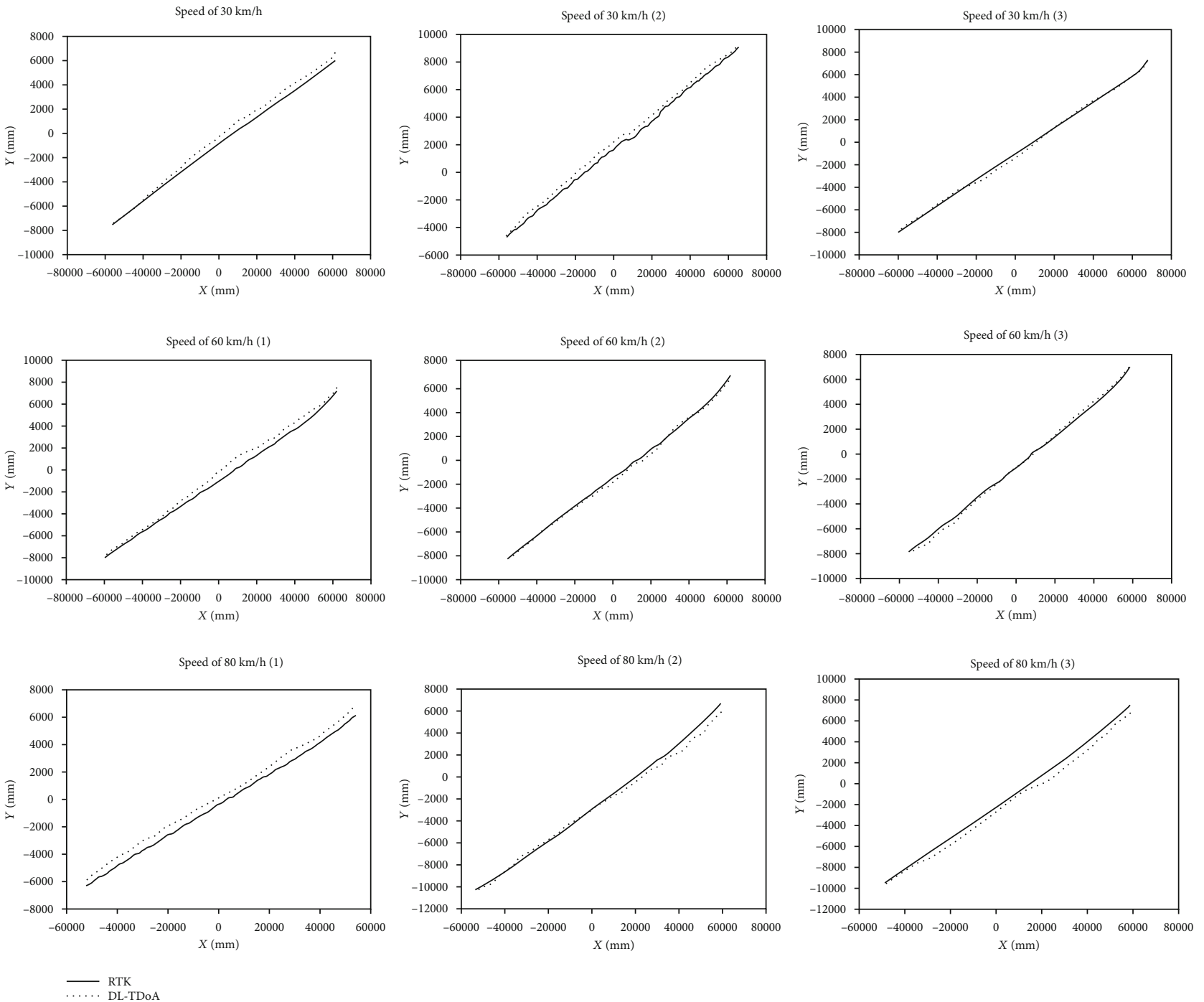

FIgUre 10: The comparisons of RTK and DL-TDoA with the speed of 30,60 , and $80 \mathrm{~km} / \mathrm{h}$, respectively.

RTK and DL-TDoA is different so testers need to manually find the same frame that both RTK and DL-TDoA have uploaded the data for the future comparisons and analysis. Figure 10 shows the comparisons of 30,60 , and $80 \mathrm{~km} / \mathrm{h}$, respectively, and the initial positions are found by testers so there might be some incorrect initial points. However, what we need to focus on is the trend of the lines, whether it is consistent with the RTK of the comparison sample. For the first and second figures of Figure 10 of $30 \mathrm{~km} / \mathrm{h}$, DL-TDoA has the same trend with RTK so the tester might not find the correct point but the results are still good. As for the third figure of Figure 10 (1), of $30 \mathrm{~km} / \mathrm{h}$, the DL-TDoA data nearly overlaps the RTK data but with a little fluctuation. That means testers find the correct initial point, and within the speed of $30 \mathrm{~km} / \mathrm{h}$, DL-TDoA performs as well as RTK. In the figures of $60 \mathrm{~km} / \mathrm{h}$ in Figure 10, (1) shows that RTK has more lag fluctuation than DL-TDoA while the other two ((2) and (3)) show the DL-TDoA performs well. As for (1) of $80 \mathrm{~km} / \mathrm{h}$ of Figure 10, it is obvious that the testers find a wrong initial position of DL-TDoA. However, compared with 30 and $60 \mathrm{~km} / \mathrm{h}$, the DL-TDoA positioning algorithm under the speed of
TABle 2: Maximum, minimum, and mean of errors of 30, 60, and $80 \mathrm{~km} / \mathrm{h}$, respectively.

\begin{tabular}{lccc}
\hline Speed $(\mathrm{km} / \mathrm{h})$ & $\begin{array}{c}\text { Maximum } \\
\text { error }(\mathrm{mm})\end{array}$ & $\begin{array}{c}\text { Minimum } \\
\text { error }(\mathrm{mm})\end{array}$ & $\begin{array}{c}\text { Mean } \\
\text { error }(\mathrm{mm})\end{array}$ \\
\hline \multirow{3}{*}{30} & 742.81 & 101.25 & 355.03 \\
& 775.68 & 117.57 & 418.85 \\
& 623.41 & 7.13 & 246.60 \\
60 & 1126.77 & 45.41 & 961.23 \\
& 647.23 & 20.96 & 302.65 \\
& 845.97 & 52.01 & 205.75 \\
80 & 1958.08 & 405.62 & 1306.81 \\
& 1496.90 & 53.26 & 951.28 \\
& 1493.73 & 62.79 & 789.23 \\
\hline
\end{tabular}

$80 \mathrm{~km} / \mathrm{h}$ performs worst. In conclusion, the higher the speed of the vehicles, the lower the accuracy of the test.

Table 2 proves that when the speed of vehicles goes up, the maximum errors and mean errors increase. However, 
TABle 3: The average mean of errors of 30,60 , and $80 \mathrm{~km} / \mathrm{h}$, respectively, and delay.

\begin{tabular}{lccc}
\hline Test scenario & Average mean error comparing with RTK $(\mathrm{cm})$ & Positioning frequency $(\mathrm{Hz})$ & Delay $(\mathrm{ms})$ \\
\hline Static & 11.96 & 10 & $<50$ \\
$30 \mathrm{~km} / \mathrm{h}$ & 37.11 & 26 & $<50$ \\
$60 \mathrm{~km} / \mathrm{h}$ & 50.06 & 26 & $<50$ \\
$80 \mathrm{~km} / \mathrm{h}$ & 87.03 & 26 & $<50$ \\
\hline
\end{tabular}
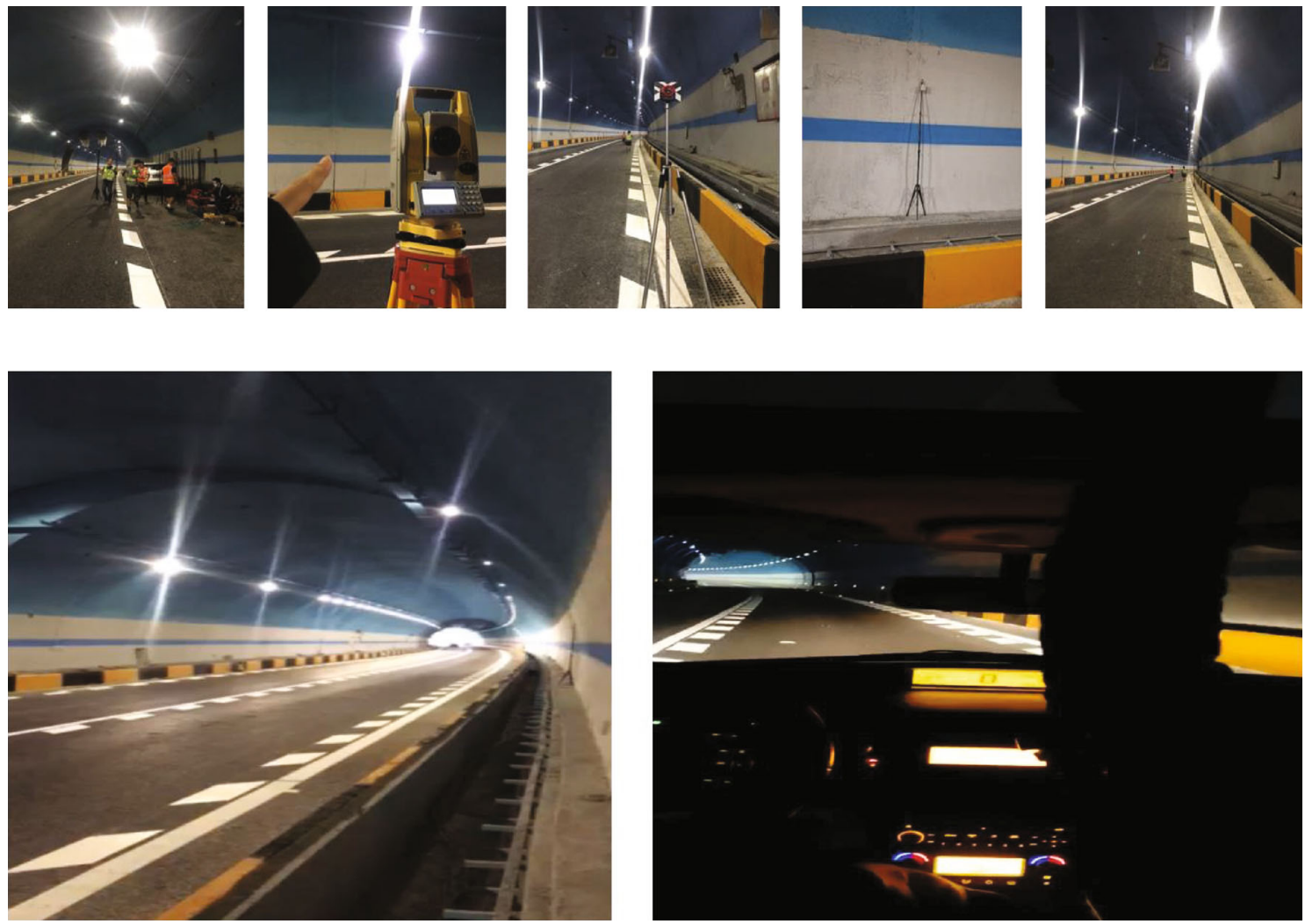

FIgURE 11: The tunnel scenario was set up.

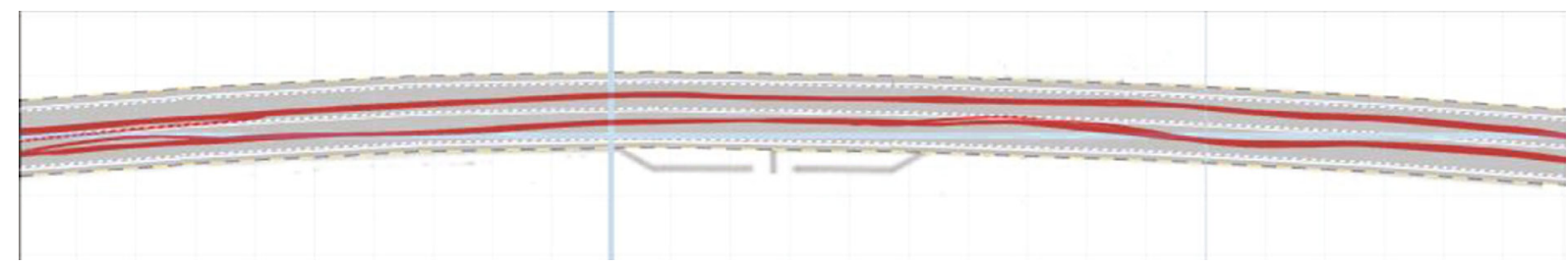

Figure 12: Tunnel test result by using DL-TDoA.

the minimum errors do not depend on speed. To ensure the safety and accurate positioning, the maximum errors are the most important data this paper needs to focus on. In conclusion in Table 3 , when the vehicle goes through the test area with 30,60 , and $80 \mathrm{~km} / \mathrm{h}$, the mean errors are $37.11,50.06$, and $87.03 \mathrm{~cm}$, respectively, which give an acceptable and useful result for future tunnel positioning without RTK comparison.

4.3. Scenario 2: Tunnel Test. In Figure 11, testers set up the scenario in a tunnel and use the same devices and sensors in the dynamic test. One driver drives the car and goes through the tunnel. Due to the unavailable RTK service, the data only comes from the DL-TDoA system. After converting the data to lines drawn on Figure 12 and checking with the real driving route, the detected trace is nearly the same accuracy as the highway dynamic test.

\section{Conclusions}

This paper proposed a DL-TDoA technology inherited from UWB wireless protocol to realize accurate vehicle positioning systems in highway and tunnel scenarios. Compared with 
traditional positioning technology, the most important advantages of DL-TDoA technology are its high time efficiency and strong anti-interference capability during data transmission. Without a cumbersome connection process, DL-TDoA utilizes simple devices and distributed processing methods to realize a reliable, large capacity, and scalable wireless network. As shown by the test results in two scenarios, regarding RTK as the standard reference position, the location accuracy from DL-TDoA is less than 1 meter and frequency keeps stable at $4 \mathrm{~Hz}, 26 \mathrm{~Hz}$. Also, the coverage rate of the DL-TDoA system achieves $100 \%$ in the tunnel scenario, which means all areas of the tunnel will be covered by the signals. In the tunnel scenario, the positioning accuracy of centimeter level can be obtained and the dynamic return can be achieved even when the vehicle system is driving at high speeds. In the future, other kinds of complex road scenes will be tested by the proposed DL-TDoA system.

\section{Data Availability}

The data can be available upon request to the corresponding author.

\section{Conflicts of Interest}

The authors declare no conflict of interest.

\section{Acknowledgments}

This research was supported by the MSIT (Ministry of Science, ICT), Korea, under the High-Potential Individuals Global Training Program (2019-0-01585 and 2020-001576) supervised by the IITP (Institute for Information \& Communications Technology Planning \& Evaluation). This research was funded by Beijing Capital Road Development Group Co., LTD., Beijing, China, and Beijing Sutong Technology Co., Ltd., Beijing, China, grant numbers “522004012-02 and 52200401-2-03." The APC was funded by Roadway Smart (Beijing) Technology Co., Ltd.

\section{References}

[1] S. Ghosh, S. Rao, and B. Venkiteswaran, "Sensor network design for smart highways," IEEE Transactions on Systems, Man, and Cybernetics - Part A: Systems and Humans, vol. 42, no. 5, pp. 1291-1300, 2012.

[2] X. Xu, J. Yu, Y. Zhu, Z. Wu, J. Li, and M. Li, "Leveraging smartphones for vehicle lane-level localization on highways," IEEE Transactions on Mobile Computing, vol. 17, no. 8, pp. 18941907, 2018.

[3] P. Varaiya, "Smart cars on smart roads: problems of control," IEEE Transactions on Automatic Control, vol. 38, no. 2, pp. 195-207, 1993.

[4] S. Wan, X. Li, Y. Xue, W. Lin, and X. Xu, "Efficient computation offloading for Internet of vehicles in edge computingassisted 5G networks," The Journal of Supercomputing, vol. 76, no. 4, pp. 2518-2547, 2020.

[5] S. Wan, R. Gu, T. Umer, K. Salah, and X. Xu, "Toward offloading Internet of vehicles applications in 5G networks," IEEE Transactions on Intelligent Transportation Systems, pp. 1-9, 2020.
[6] C. Liu, K. T. Chau, D. Wu, and S. Gao, "Opportunities and challenges of vehicle-to-home, vehicle-to-vehicle, and vehicle-to-grid technologies," Proceedings of the IEEE, vol. 101, no. 11, pp. 2409-2427, 2013.

[7] S. Zhou and J. K. Pollard, "Position measurement using Bluetooth," IEEE Transactions on Consumer Electronics, vol. 52, no. 2, pp. 555-558, 2006.

[8] F. Forno, G. Malnati, and G. Portelli, "Design and implementation of a Bluetooth ad hoc network for indoor positioning," IEE Proceedings - Software, vol. 152, no. 5, pp. 223-228, 2005.

[9] Y. Tao and L. Zhao, "A novel system for WiFi radio map automatic adaptation and indoor positioning," IEEE Transactions on Vehicular Technology, vol. 67, no. 11, pp. 10683-10692, 2018.

[10] C. Y. Yao and W. C. Hsia, "An indoor positioning system based on the dual-channel passive RFID technology," IEEE Sensors Journal, vol. 18, no. 11, pp. 4654-4663, 2018.

[11] S. H. Fang, C. H. Wang, T. Y. Huang, C. H. Yang, and Y. S. Chen, "An enhanced ZigBee indoor positioning system with an ensemble approach," IEEE Communications Letters, vol. 16, no. 4, pp. 564-567, 2012.

[12] Z. Yin, X. Jiang, Z. Yang, N. Zhao, and Y. Chen, "WUB-IP: a high-precision UWB positioning scheme for indoor multiuser applications," IEEE Systems Journal, vol. 13, no. 1, pp. 279288, 2019.

[13] X. Shan and Z. Shen, "Miniaturized UHF/UWB tag antenna for indoor positioning systems," IEEE Antennas and Wireless Propagation Letters, vol. 18, no. 12, pp. 2453-2457, 2019.

[14] M. Eric and D. Vucic, "Method for direct position estimation in UWB systems," Electronics Letters, vol. 44, no. 11, pp. 701-703, 2008.

[15] M. Hamalainen, J. Iinatti, V. Hovinen, and M. Latva-aho, "Inband interference of three kinds of UWB signals in GPS L1 band and GSM900 uplink band," in 12th IEEE International Symposium on Personal, Indoor and Mobile Radio Communications. PIMRC 2001. Proceedings (Cat. No.01TH8598), San Diego, CA, USA, 2001.

[16] A. Zhou, S. Wang, S. Wan, and L. Qi, "LMM: latency-aware micro-service mashup in mobile edge computing environment," Neural Computing and Applications, vol. 32, no. 19, pp. 15411-15425, 2020.

[17] X. Xu, B. Shen, X. Yin et al., "Edge server quantification and placement for offloading social media services in industrial cognitive IoV," IEEE Transactions on Industrial Informatics, p. $1,2020$.

[18] E. Wang, Z. Zhang, and M. Cai, "A study on BeiDou positioning performance analysis platform based on LabVIEW," in 2013 IEEE 8th Conference on Industrial Electronics and Applications (ICIEA), pp. 963-967, Melbourne, VIC, Australia, 2013.

[19] D. V. Nguyen, F. Nashashibi, T. K. Dao, and E. Castelli, "Improving poor GPS area localization for intelligent vehicles," in 2017 IEEE International Conference on Multisensor Fusion and Integration for Intelligent Systems (MFI), pp. 417-421, Daegu, South Korea, November 2017.

[20] A. Bhaskar, M. Qu, and E. Chung, "Bluetooth vehicle trajectory by fusing Bluetooth and loops: motorway travel time statistics," IEEE Transactions on Intelligent Transportation Systems, vol. 16, no. 1, pp. 113-122, 2015.

[21] W. Albazrqaoe, J. Huang, and G. Xing, "A practical Bluetooth traffic sniffing system: design, implementation, and 
countermeasure," IEEE/ACM Transactions on Networking, vol. 27, no. 1, pp. 71-84, 2019.

[22] B. Song, J. Y. Hwang, and K. A. Shim, "Security improvement of an RFID security protocol of ISO/IEC WD 29167-6," IEEE Communications Letters, vol. 15, no. 12, pp. 1375-1377, 2011.

[23] Y. Y. Shih, W. H. Chung, P. C. Hsiu, and A. C. Pang, "A mobility-aware node deployment and tree construction framework for ZigBee wireless networks," IEEE Transactions on Vehicular Technology, vol. 62, no. 6, pp. 2763-2779, 2013.

[24] J. F. Gerrits, J. R. Farserotu, and J. R. Long, "Low-complexity ultra-wide-band communications," IEEE Transactions on Circuits and Systems II: Express Briefs, vol. 55, no. 4, pp. 329-333, 2008.

[25] P. du, S. Zhang, C. Chen, A. Alphones, and W. D. Zhong, "Demonstration of a low-complexity indoor visible light positioning system using an enhanced TDOA scheme," IEEE Photonics Journal, vol. 10, no. 4, pp. 1-10, 2018.

[26] J. Wang, X. Qiu, and Y. Tu, “An improved MDS-MAP localization algorithm based on weighted clustering and heuristic merging for anisotropic wireless networks with energy holes," Computers, Materials \& Continua, vol. 60, no. 1, pp. 227-244, 2019.

[27] I. Um, S. Park, H. T. Kim, and H. Kim, "Configuring RTK-GPS architecture for system redundancy in multi-drone operations," IEEE Access, vol. 8, pp. 76228-76242, 2020.

[28] K. M. Ng J. Johari et al., "Performance evaluation of the RTKGNSS navigating under different landscape," in 2018 18th International Conference on Control, Automation and Systems (ICCAS), pp. 1424-1428, Daegwallyeong, 2018. 